\title{
A Study on Driving Simulation and Efficiency Maps with Nonlinear IPMSM Datasets
}

\author{
Won-Ho Kim ${ }^{1}$, Ik-Sang Jang ${ }^{1}$, Ki-Doek Lee ${ }^{1}$, Jong-Bin Im ${ }^{1}$, Chang-Sung Jin ${ }^{2}$, Dae-Hyun Koo ${ }^{3}$, and Ju Lee ${ }^{1}$ * \\ ${ }^{1}$ Hanyang University, Haengdang-dong, Seongdong-gu, Seoul 133-791, Korea \\ ${ }^{2}$ Samsung Techwin, 701 Sampyeong-dong, Bundang-gu, Seongnam-Si 463-400, Korea \\ ${ }^{3}$ Korea Electrotechnology Research Institute, Changwon Si 642-120, Korea
}

(Received 30 September 2010, Received in final form 28 January 2011, Accepted 28 January 2011)

\begin{abstract}
Hybrid electric vehicles have attracted much attention of late, emphasizing the necessity of developing traction motors with a high input current and a wide speed range. Among such traction motors, various researches have been conducted on interior permanent-magnet synchronous motors (IPMSMs) with high power density and mechanical solidity. Due to the complexity of its parameters, however, with nonlinear motor characteristics and current vector control, it is actually difficult to accurately estimate the base speed within an actual operating speed range or a voltage limit. Moreover, it is impossible to construct an efficiency map as the efficiency differs according to the control mode. In this study, a simulation method for operation performance considering the nonlinearity of IPMSM was proposed. For this, datasets of various nonlinear parameters were made via the finite-element method and interpolation. Maximum torque-per-ampere and flux-weakening control were accurately simulated using the datasets, and an IPMSM efficiency map was accurately constructed based on the simulation. Lastly, the validity of the simulation was verified through tests.
\end{abstract}

Keywords : IPMSM (interior permanent-magnet synchronous motor), MTPA (maximum torque-per-ampere) control, FW (flux-weakening) control, efficiency map

\section{Driving Simulation Considering the Control Mode}

Most interior permanent-magnet synchronous motors (IPMSMs) use maximum power control, which gains the maximum power within the current and voltage limit [1]. At present, maximum torque-per-ampere (MTPA) control is used considering only the current limit, up to the base speed, at which the input voltage reaches the voltage limit. In areas that show a speed higher than the base speed, flux-weakening (FW) control is used to reduce the induced voltage by increasing the d-axis current. The flowchart of the driving simulation proposed in this study is shown in Fig. 1.

\subsection{MTPA Control}

First, after fixing the rotating speed, the 2D-torque dataset per current phase angle is calculated using equation (1). At this time, the 2D-inductance dataset calculated via

*Corresponding author: Tel: +82-2-2220-0342

Fax: +82-2-2295-7111, e-mail: julee@hanyang.ac.kr the finite-element method (FEM) and flux linkage at the no-load condition is used.

$$
\left\lfloor\mathrm{T}_{\text {total }}\right\rfloor=\mathrm{P}_{\mathrm{n}} \Psi_{\mathrm{a}} \mathrm{I}_{\mathrm{a}} \cos \beta+\frac{1}{2}\left(\left\lfloor\mathrm{~L}_{\mathrm{q}}\right\rfloor-\left\lfloor\mathrm{L}_{\mathrm{d}}\right\rfloor\right) \mathrm{I}_{\mathrm{a}}^{2} \sin 2 \beta
$$

Finally, various data showing the driving characteristics are calculated using the determined current phase angle and rotating speed. Moreover, if the maximum value is lower than the voltage limit after the calculation of the 2D-input voltage dataset, the fixed rotating speed is increased, and MTPA control is performed again.

\subsection{FW Control}

As shown in Fig. 2, a current phase angle shall be calculated through an intersection point between a voltage limit ellipse and a current limit circle. The utilization of the generally used induced voltage makes the calculation of an intersection point (P1) simple. When using the input voltage, however, it is impossible to find point $\mathrm{P} 2$ without numerical calculation. In this study, two intersection points were sought using the secant method. The characteristics that were determined through these processes are shown 


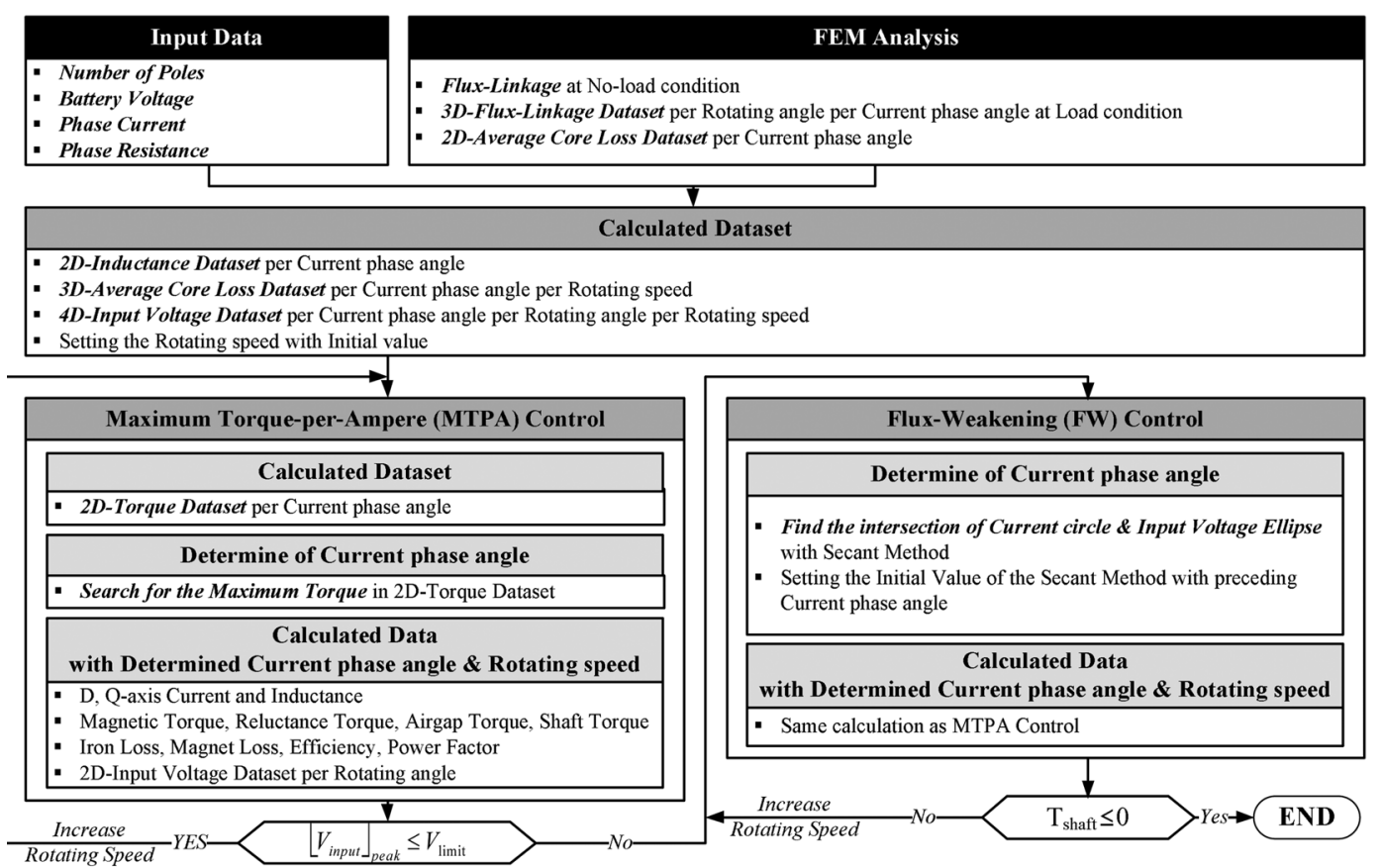

Fig. 1. Driving simulation process for IPMSM.
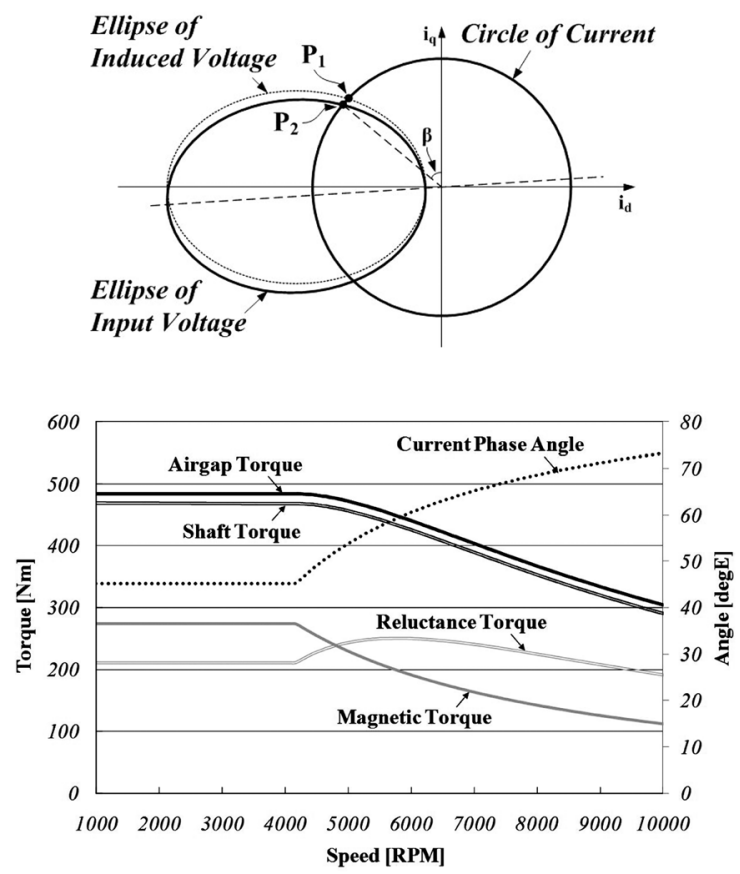

Fig. 2. Voltage and current limit and the results of the driving simulation.

in Fig. 2.

\section{Construction of an Efficiency Map via Driving Simulation}

To be able to construct an efficiency map, the driving
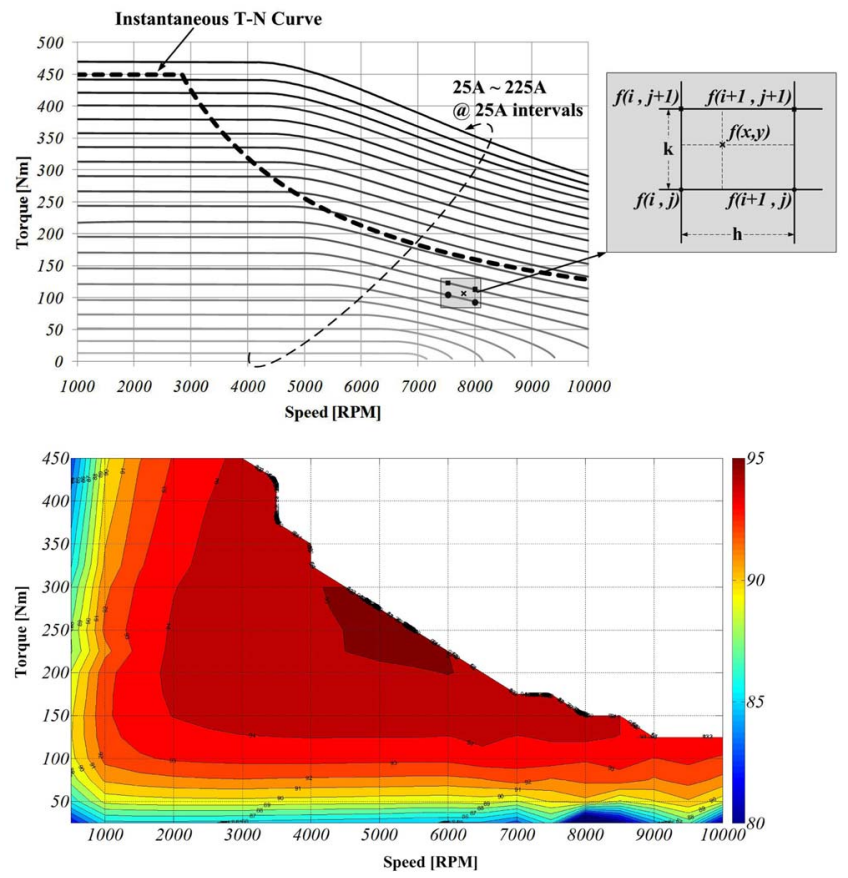

Fig. 3. T-N curve for the variable currents (left) and the efficiency map (right).

characteristics within the target driving range must be understood. In this study, as shown in Fig. 3, driving simulation was performed with a $25 \mathrm{~A}$ current increase, and the loss data were included. The loss of a point that did not pass over a driving simulation line was calculated by applying successive univariate interpolating polyno- 


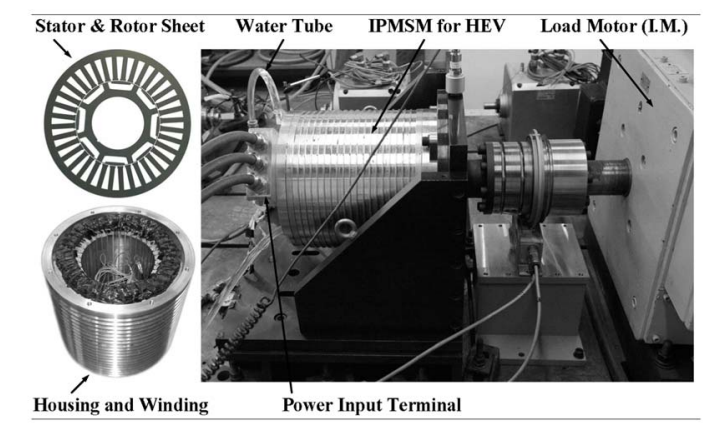

\begin{tabular}{ccc}
\hline \hline Speed / Current & $\begin{array}{c}\text { Torque [Nm] /Efficiency [\%] } \\
\text { [sim. vs Exp.] }\end{array}$ & $\begin{array}{c}\text { Error Ratio [\%] } \\
\text { [Tor. / Eff.] }\end{array}$ \\
\hline 1000RPM / 168A & $218 / 93.6$ vs 221/94.3 & $98.6 / 99.3$ \\
2843RPM / 168A & $217 / 95.8$ vs 221/95.7 & $98.2 / 100.1$ \\
5000RPM / 108A & $132 / 96.2$ vs $123 / 94.6$ & $107.3 / 101.7$ \\
7000RPM / 84A & $90 / 95.9$ vs $94 / 94.6$ & $95.7 / 101.4$ \\
\hline \hline
\end{tabular}

Fig. 4. Experiment equipment and the results of the simulation and experiment.

mials, and is shown in Fig. 3. This resulted from the application of Newton's forward difference interpolation to the $\mathrm{x}$ and $\mathrm{y}$ axes for two successive times. The efficiency map constructed via driving simulation is shown in Fig. 3.

\section{Verification through a Test}

Fig. 4 shows the setup of the motor and test equipment as well as the comparison data between the characteristics in the torque and the efficiency of a test value and an analyzed value. It was found to be proper as the error rate was below $5 \%$.

\section{Acknowledgement}

This work was supported by the National Research Foundation of Korea (NRF) grant funded by the Korea government (MEST) (No. 2008-0060145).

\section{References}

[1] S. Morimoto, Y. Takeda, and T. Hirasa, IEEE Trans. Power Electron, 5, 133 (1990).

[2] Sung-Il Kim, Geun-Ho Lee, Jung-Pyo Hong, and Tae-Uk Jung, IEEE Trans. Magn. 44, 1590 (2008).

[3] Rich Schiferl, Design Considerations for Salient Pole, PM Synchronous Motors in Variable Speed Drive Applications, Ph. D. Thesis, University of Wisconsin-Madison (1987).

[4] K. C. Kim, S. B. Lim, D. H. Koo, and J. Lee, IEEE Trans. Magn. 42, 3485 (2006). 